\title{
Prognostic factors and patterns of recurrence among patients treated for endometrial cancer in Southern Brazil
}

\author{
G.A. Carvalho' ${ }^{1,2}$, M.P. Salcedo ${ }^{1,3}$, S.A. Pessini ${ }^{1,4}$ \\ ${ }_{1}^{1}$ Programa de Pós Graduação em Ginecologia e Obstetrícia da Universidade Federal do Rio Grande do Sul (PPG-GO UFRGS). \\ ${ }^{2}$ Hospital Escola da Universidade Federal de Pelotas (HE-UFPEL), Pelotas, Brazil. \\ 3 Universidade Federal de Ciências da Saúde de Porto Alegre (UFCSPA) / Irmandade Santa Casa de Misericórdia de Porto \\ Alegre (ISCMPA), Porto Alegre, Brazil. \\ ${ }^{4}$ Universidade Federal do Rio Grande do Sul (UFRGS), Porto Alegre, Brazil.
}

\section{Background:}

- Endometrial cancer is the first most common cancer in female genital tract in Western countries.

- In spite of having a higher incidence in developed nations, the mortality continues superior in low and middle-income countries as Brazil.

\section{Objectives:}

The goal of our study was:

- To compare clinical and histopathological characteristics of women with and without recurrence of endometrial cancer after primary surgical treatment.

\section{Methods:}

- A retrospective cohort study was carried out in ISCMPA with patients treated for endometrial cancer from 1995 to 2016, in Southern Brazil;

- The variables analyzed were: age, symptom and method for the diagnosis, menopausal status, body mass index (BMI), comorbidities, histopathological type, FIGO staging and recurrence rate in the sample;

- Cox regression was used to identify independent prognostic factors;

- Overall survival was calculated by using the Kaplan-Meier method.

\section{Results:}

Of the 216 women underwent surgical treatment for endometrial carcinoma and met the inclusion criteria for the study, 23 (10.6\%) have developed recurrent disease after a mean time of 3.7 years $(S D \pm 4.2)$.
- Depth of myometrial infiltration, stromal cervical involvement, FIGO staging and death were variables with a significant difference between patients with and without recurrence.

- In women with cancer recurrence, $60.9 \%$ died, compared to $21.6 \%$ of those without recurrence ( $p$ $\leq 0.0001$, Chi-square test).

- Disease free survival was significant different between women with and without recurrence ( $p$ $\leq 0.0001$, log-rank test).

\section{Conclusions:}

- This study ratifies depth of myometrial infiltration greater than $50 \%$, cervical stromal invasion and FIGO staging are important prognostic factors to endometrial cancer recurrence.

- The recurrence of this tumor is related to a reduction in overall survival.

- These data are compatible with the existing literature and complement the scarce data on endometrial cancer in Brazil.

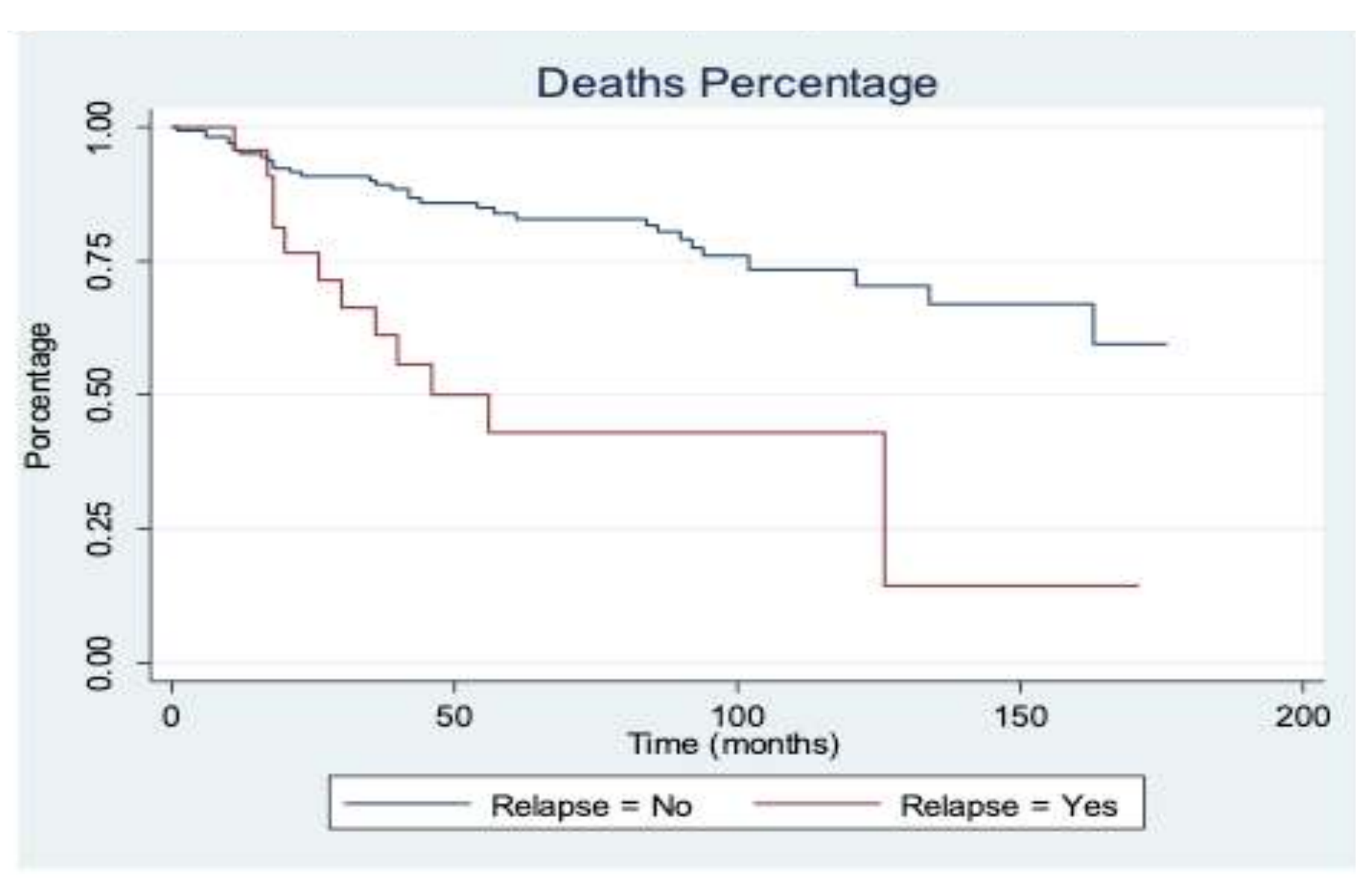

Figure 1. Kaplan Meier survivor estimates for patients with and without relapse. 